\title{
25. MINOR ELEMENT GEOCHEMISTRY OF SEDIMENTS AT SITE 328, FALKLAND OUTER BASIN AND SITE 329, FALKLAND PLATEAU, LEG 36, DEEP SEA DRILLING PROJECT
}

\author{
J. Tarney and N.C.B. Donnellan, Department of Geological Sciences, \\ University of Birmingham, England
}

\begin{abstract}
Site 328, a deep-water site in the Malvinas Outer Basin, is composed of siliceous and zeolitic clays and claystones. Site 329, an equivalent shallow-water site on the nearby Falkland Plateau, is composed of siliceous and calcareous oozes and chalks deposited mostly above the CCD. A total of 72 samples from Site 328 and 74 from Site 329 has been analyzed for Ti, Fe, Mn, Cr, Ni, Cu, Zn, Rb, $\mathrm{Sr}, \mathrm{Y}, \mathrm{Zr}, \mathrm{Nb}, \mathrm{Ba}, \mathrm{La}, \mathrm{Ce}, \mathrm{Pb}$, and $\mathrm{Th}$ in order to establish in detail the downhole geochemical variations at each site and to compare the geochemical behavior of trace elements in a deep water and a shallow water environment.

There are significant short, medium-, and long-term geochemical variations at both sites, but particularly at Site 329 , and wellmarked sympathetic and antipathetic relationships between many of the minor and trace elements. Many of the downhole variations correlate with lithology and stratigraphy. Geochemically the two sites are very different, not only in terms of mean element compositions, but also in the way the trace elements interrelate. These differences are examined in detail by use of correlation coefficient matrices. The results emphasize the fact that the sediment sources and depositional environments were fundamentally different at the two sites.
\end{abstract}

\section{INTRODUCTION}

There were several reasons for undertaking geochemical studies of the sediments at Sites 328 and 329:

1) To compare the chemistry of sediments of approximately the same age range in two adjacent high latitude sites, one (328) deposited at great depth well below the carbonate compensation depth (CCD) and the other (329) deposited in a shelf environment on the Falkland Plateau mostly just above the CCD.

2) To assess whether there was any significant variation in the chemistry of the sediments with depth in the core. At both sites there was, for paleontological reasons, some continuous coring. As far as possible, to give close coverage, samples were taken every section. A related objective was to establish the degree of smallscale chemical variability in these continuously cored sections.

3) To establish whether there was any change in chemistry which might correlate with lithology and with change in depositional environments.

4) At Site 328 siliceous organisms showed increasing dissolution below Unit 2 (Miocene) and were replaced lower down the core by zeolitic clays and claystones. There was some shipboard interest as to whether this might be reflected in a major change in trace element chemistry.

\section{LOCATION AND SAMPLING}

Site 328 was located in the Malvinas Outer Basin (lat $49^{\circ} 48.67^{\prime} \mathrm{S}$; long $36^{\circ} 39.53^{\prime} \mathrm{W}$ ) in a water depth of 5103 meters. The sediments were drilled to a depth of 471 meters, only an estimated 90 meters above the acoustic basement. The top 50 meters consist of Plio-Pleistocene siliceous ooze, underlain by Oligocene and Miocene silty siliceous and zeolitic clay. There is a sharp change below 50 meters (mid-Oligocene) to a rather monotonous greenish-gray zeolitic clay which constitutes the remainder of the section and ranges in age down to Upper Cretaceous (Turonian).

Two of the three holes at this site were sampled. Hole 328 cored intermittently through the succession to a depth of 400 meters. Hole $328 \mathrm{~B}$ cored continuously the uppermost 65 meters from Pleistocene through Pliocene, Miocene, Oligocene to reach uppermost Eocene, with further penetration (Core 7) at 450 meters in Late Cretaceous claystones.

Site 329, located on the elevated part of the Falkland Plateau (lat $50^{\circ} 39.21^{\prime} \mathrm{S}$; long $46^{\circ} 05.73^{\prime} \mathrm{W}$ ) in a water depth of 1530 meters, penetrated 464 meters of shallow water siliceous and calcareous oozes and chalks. The hole was cored continuously for almost 180 meters (Cores 1 to 19), and intermittently thereafter. The upper 4 meters consisted of Pleistocene silty clay-rich diatomaceous oozes, but the bulk of the section ( $375 \mathrm{~m})$ was made up of Miocene nanno and diatom oozes overlying Oligocene to Paleocene nanno chalk. Variations in the proportion of diatom and nanno components throughout most of the core suggest that deposition took place near to, but mostly above the CCD.

Sampling at both sites was carried out onboard ship. As far as possible every core and most of the sections 
within each core were sampled, except where there had been severe drilling disturbance of the core. About 30$40 \mathrm{~g}$ of each sediment sample was taken, and stored in a sealed polythene vial prior to analysis.

A total of 146 samples was selected for analysis, 42 from Hole 328, 30 from Hole 328B, and 74 from Hole 329.

With the large number of samples selected for analysis, fairly rapid methods had to be employed in preparing the samples for analysis by X-ray fluorescence techniques. Nevertheless time spent in sample preparation far exceeded the time spent on the analysis itself.

The samples were oven-dried at $110^{\circ} \mathrm{C}$ for several hours in a disaggregated state; crushed in a tungsten carbide ball mill for $20 \mathrm{~min}$, and pressed into a $4.6-\mathrm{cm}$ diameter disc at 20 tons using a small amount of MOWIOL as a binder. The powder discs were then analyzed for $\mathrm{Ti}, \mathrm{Fe}, \mathrm{Mn}, \mathrm{Cr}, \mathrm{Ni}, \mathrm{Cu}, \mathrm{Zn}, \mathrm{Rb}, \mathrm{Sr}, \mathrm{Y}, \mathrm{Zr}$, $\mathrm{Nb}, \mathrm{Ce}, \mathrm{La}, \mathrm{Pb}$, and Th using an automatic Philips PW $1450 \mathrm{X}$-ray spectrometer. Calibration was based on international standard rocks and "spiked" samples. The trace element results were automatically corrected for interelement absorption effects using Mo Compton scatter and WL $\beta$ tube lines. Precision is better than \pm 2 ppm for most trace elements.

Light elements (i.e., most major elements) were not analyzed for at this stage because seawater salt, coating the grains, had a serious absorption effect on the longer wavelengths. Ideally, each sample needs to be thoroughly washed with distilled water before drying in order to overcome this problem, but this would considerably extend the preparation time.

\section{RESULTS}

Analytical results for Hole 328, Hole 328B, and Hole 329 are given in Tables 1, 2, and 3, respectively, and are arranged in downhole order. Mean values for the siliceous clay sediments at Site 328 and the siliceous calcareous sediments at Site 329 are compared in Table 4. Also listed in Table 4 are the maximum and minimum values and the standard deviation for each element and element ratio. As may be expected, there are substantial differences in the mean values of most elements between the two sites. This is also shown in Table 4 as a ratio of Site 328/Site 329 for each element. All the elements studied have a higher concentration in the Site 328 clay section apart from $\mathrm{Sr}$ and to a smaller extent $\mathrm{Ba}$.

Before comparing the geochemistry of the sediments at the two sites, it is necessary to discuss geochemical variations within each site individually.

\section{Site 329}

Downhole element variations at Site 329 are shown diagrammatically in Figure 1. To provide a compact picture of the geochemical variations on one diagram, the samples are plotted incrementally in downhole order without regard as to whether the cores were continuous or not, or even whether all individual sections within each core were represented. Hence the vertical scale is nonlinear. Nevertheless, variations in chemistry with depth in the core are clearly brought out.

Several interesting features are apparent from this diagram.

1) There are both sympathetic and antipathetic relationships between the different elements in the sediments. The variation pattern for $\mathrm{Zn}$ is almost the identical mirror image of that for $\mathrm{Sr}$, indicating that $\mathrm{Zn}$ is associated with one major component of the sediment while $\mathrm{Sr}$ is associated with the other. That $\mathrm{Sr}$ is linked with the calcareous component can be seen from the pattern of the plotted shipboard $\mathrm{CaCO}_{3}$ results which, although there are fewer determinations and they were not taken from the same sections of the cores, show variations comparable to that of Sr. Most of the other elements figured have variations sympathetic to a greater or lesser degree with $\mathrm{Zn}$. Of these $\mathrm{Cr}, \mathrm{Ni} \mathrm{Rb}$, $\mathrm{Th}, \mathrm{Pb}$, and $\mathrm{Ba}$ correlate strongly, while $\mathrm{Zr}, \mathrm{Nb}, \mathrm{Y}, \mathrm{Ce}$ and $\mathrm{La}$ show comparable variations but of smaller magnitude. Ba follows $\mathrm{Zn}$ very closely in the oozes of the upper part of the core, but rather less closely in the chalks near the bottom of the hole.

2) Short-, medium-, and long-term trends are evident in the element abundance levels in the sediments. Because the precision of XRF determinations for the elements studied is of the order of 1 to $2 \mathrm{ppm}$, these trends appear to be real. In the continuously cored section (Cores 1 to 19) smooth trends are evident (e.g., Sr, $\mathrm{Rb}$ ) extending over several cores. Even in the discontinuously cored zones some smooth trends are present. Systematic long-term variations are seen within the medium-term scatter. For instance, $\mathrm{Sr}$ increases from $130 \mathrm{ppm}$ to nearly $200 \mathrm{ppm}$ between Core 4 and Core 17 , while $\mathrm{Zn}$ decreases from $120 \mathrm{ppm}$ to $20 \mathrm{ppm}$ over the same interval. Again $\mathrm{Ba}$ increases from $200 \mathrm{ppm}$ in Core 17 to $800 \mathrm{ppm}$ in Core 33. Comparable long-term variations are seen for some other elements, while others show no long-term variation but do show sympathetic medium- to short-term variation. Significant short-term peaks for many elements are seen in the upper part of Core 2, in Core 8, and in Core 13. Some of the apparent short-term variation in the lower part of the core, however, may just be a result of discontinuous coring.

3) Some geochemical variations are directly related to lithology and stratigraphy. Hole 329 consists mainly of diatom-nanno oozes and chalks with variations in the proportion of diatom and calcareous nanno components, but with variations in the radiolarian and clay components too.

The sharp geochemical discontinuity between the upper and lower halves of Core 1, affecting every element, coincides with the hiatus between Pleistocene and Miocene. Higher trace-element concentrations in the Pleistocene are linked with the high proportion of clay (glauconite) and sand. The upper two sections of Core 2 have a similar geochemistry and lithology and may simply be a result of downhole contamination with Pleistocene sand.

In Core 4 there is a progressive change from diatombearing nanno ooze in the upper sections to a nannoand clay-bearing diatom ooze in the lower part, and 
TABLE 1

Geochemical Data for Siliceous Clays, Hole 328, Malvinas Outer Basin (oxides in \%, trace elements in ppm)

\begin{tabular}{|c|c|c|c|c|c|c|c|c|c|c|c|c|c|c|c|c|c|}
\hline $\begin{array}{c}\text { Sample } \\
\text { (Interval in cm) }\end{array}$ & $\mathrm{TiO}_{2}$ & $\mathrm{Fe}_{2} \mathrm{O}_{3}$ & $\mathrm{MnO}$ & $\mathrm{Cr}$ & $\mathrm{Ni}$ & $\mathrm{Cu}$ & $\mathrm{Zn}$ & $\mathrm{Rb}$ & $\mathrm{Sr}$ & $\mathrm{Y}$ & $\mathrm{Zr}$ & $\mathrm{Nb}$ & $\mathrm{Ba}$ & $\mathrm{La}$ & $\mathrm{Ce}$ & $\mathrm{Pb}$ & Th \\
\hline $1-1,140$ & 0.604 & 5.31 & 0.136 & 48 & 66 & 283 & 112 & 74 & 131 & 30 & 151 & 8 & 506 & 28 & 40 & 11 & 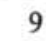 \\
\hline $1-2,135$ & - & - & - & 46 & 81 & - & 126 & 77 & 146 & 19 & 154 & 6 & 526 & 24 & 44 & 12 & 9 \\
\hline $1-3,124$ & - & - & - & 59 & 63 & 223 & 137 & 90 & 146 & 20 & 155 & 11 & 674 & 24 & 41 & 20 & 10 \\
\hline $1-4,127$ & 0.716 & 6.12 & 0.121 & 58 & 53 & 281 & 135 & 94 & 157 & 23 & 169 & 14 & 470 & 28 & 45 & 18 & 10 \\
\hline $1-5,127$ & 0.622 & 4.99 & 0.112 & 50 & 110 & 355 & 126 & 64 & 132 & 15 & 141 & 11 & 620 & 17 & 43 & 13 & 8 \\
\hline $2-2,135$ & - & - & - & 35 & 62 & - & 99 & 71 & 150 & 19 & 153 & 9 & 533 & 27 & 40 & 12 & 10 \\
\hline $2-3,125$ & 0.410 & 5.46 & 1.789 & 30 & 300 & 488 & 125 & 81 & 157 & 19 & 157 & 10 & 443 & 22 & 33 & 15 & 5 \\
\hline $2-4,136$ & 0.576 & 6.19 & 0.250 & 39 & 57 & 346 & 125 & 92 & 160 & 20 & 160 & 10 & 431 & 23 & 36 & 13 & 10 \\
\hline $2-5,136$ & 0.475 & 5.19 & 0.117 & 37 & 40 & 322 & 117 & 89 & 157 & 20 & .202 & 11 & 384 & 23 & 36 & 10 & 9 \\
\hline $2-6,55$ & 0.487 & 5.14 & 0.114 & 37 & 46 & 329 & 124 & 81 & 145 & 16 & 151 & 9 & 284 & 21 & 30 & 10 & 9 \\
\hline $3-1,126$ & 0.488 & 4.65 & 0.164 & 43 & 61 & 431 & 143 & 99 & 161 & 20 & 174 & 11 & 544 & 31 & 49 & 10 & 9 \\
\hline $3-3,131$ & 0.845 & 7.47 & 0.516 & 39 & 198 & 547 & 171 & 101 & 165 & 25 & 188 & 13 & 726 & 34 & 55 & 22 & 10 \\
\hline $3-4,131$ & 0.770 & 7.36 & 0.296 & 41 & 154 & 603 & 175 & 105 & 176 & 27 & 198 & 12 & 1077 & 37 & 54 & 12 & 10 \\
\hline $3-5,115$ & 0.734 & 7.08 & 0.287 & 34 & 116 & 533 & 196 & 96 & 171 & 30 & 199 & 11 & 1028 & 33 & 52 & 16 & 10 \\
\hline $3-6,74$ & 0.814 & 7.59 & 0.278 & - & - & 451 & 181 & 85 & 165 & 29 & - & - & 1007 & - & - & 18 & 12 \\
\hline $4-2,130$ & - & - & - & 57 & 137 & 276 & 172 & 111 & 114 & 23 & 145 & 10 & 308 & 40 & 63 & 15 & 13 \\
\hline $4-3,104$ & 0.682 & 6.88 & 0.571 & 79 & 169 & 323 & 184 & 110 & 128 & 24 & 15 & 11 & 341 & 30 & 62 & 14 & 11 \\
\hline $4-5,131$ & 0.981 & 9.08 & 0.435 & 54 & 125 & 227 & 200 & 111 & 131 & 29 & 174 & 13 & 427 & 43 & 101 & 25 & 19 \\
\hline $5-2,84$ & 0.567 & 6.54 & 0.094 & 76 & 105 & - & 143 & 110 & 115 & 25 & 130 & 12 & 523 & 44 & 83 & 20 & 17 \\
\hline $5-3,133$ & - & - & - & 907 & 399 & - & 153 & 110 & 120 & 24 & 132 & 8 & 478 & 25 & 45 & 27 & 17 \\
\hline $6-1,103$ & 0.802 & 7.93 & 0.09 & 70 & 40 & 254 & 179 & 101 & 113 & 25 & 139 & 12 & 420 & 41 & 95 & 30 & 16 \\
\hline $6-2,123$ & 0.707 & 8.66 & 0.107 & 63 & 66 & 259 & 118 & 94 & 109 & 22 & 13 & 11 & 354 & 42 & 79 & 27 & 14 \\
\hline $6-3,62$ & 0.685 & 7.23 & 0.118 & 64 & 115 & 268 & 139 & 94 & 151 & 32 & 133 & 13 & 592 & 43 & 85 & 26 & 17 \\
\hline $7-4,138$ & 0.784 & 8.41 & 0.103 & 80 & 60 & 239 & 117 & 102 & 115 & 26 & 135 & 13 & 366 & 44 & 91 & 26 & 15 \\
\hline $8-2,138$ & 0.788 & 9.97 & 0.107 & 70 & 63 & 197 & 130 & 101 & 125 & 23 & 127 & 11 & 537 & 37 & 90 & 15 & 16 \\
\hline $8-3,38$ & 0.824 & 9.80 & 0.1 & 74 & 68 & 213 & 139 & 98 & 120 & 23 & 138 & 10 & 341 & 44 & 96 & 16 & 15 \\
\hline $9-1,100$ & 0.793 & 8.03 & 0.10 & 67 & 62 & 264 & 137 & 101 & 126 & 24 & 137 & 12 & 512 & 47 & 87 & 27 & 16 \\
\hline $9-2,33$ & 0.807 & 9.48 & 0.120 & 74 & 69 & 238 & 143 & 99 & 117 & 24 & 136 & 11 & 397 & 36 & 90 & 17 & 15 \\
\hline $9-4,78$ & 0.843 & 9.33 & 0.107 & 77 & 77 & 215 & 138 & 102 & 126 & 25 & 140 & 12 & 631 & 42 & 92 & 22 & 17 \\
\hline $9-5,100$ & 0.840 & 7.31 & 0.1 & 80 & 79 & 300 & 149 & 101 & 134 & 25 & 15 & 14 & 478 & 42 & 95 & 31 & 19 \\
\hline $9-6,108$ & 0.792 & 8.0 & 0 & 69 & 71 & 25 & 137 & 106 & 129 & 23 & 14 & 10 & 580 & 40 & 80 & 23 & 16 \\
\hline $10-1,42$ & 0.743 & 10.21 & 0.1 & 60 & 40 & 197 & 109 & 105 & 125 & 31 & 134 & 10 & 733 & 43 & 79 & 12 & 16 \\
\hline $10-5,124$ & 0.703 & 11.09 & 0.133 & 45 & 53 & 172 & 122 & 104 & 119 & 33 & 133 & 11 & 295 & 42 & 90 & 15 & 13 \\
\hline $10-6,125$ & 0.764 & 6.25 & 0.106 & 61 & 82 & 260 & 179 & 99 & 146 & 36 & 406 & 32 & 504 & 53 & 126 & 23 & 19 \\
\hline $11-1,3$ & 0.771 & .27 & 0.1 & 56 & 61 & 217 & 112 & 78 & 127 & 28 & 15 & 13 & 412 & 43 & 93 & 17 & 11 \\
\hline $11-3,102$ & 0.467 & 6.46 & 0 & 63 & 72 & 26 & 145 & 87 & 13 & 27 & 1 & 11 & 415 & 42 & 88 & 32 & 15 \\
\hline $11-4,77$ & 0.209 & 3.79 & 0.104 & 1089 & 266 & 169 & 120 & 88 & 129 & 23 & 117 & 10 & 460 & 24 & 49 & 15 & 10 \\
\hline $11-5,65$ & 0.432 & 8.97 & 0.101 & 53 & 61 & 182 & 96 & 82 & 122 & 22 & 138 & 12 & 355 & 42 & 88 & 12 & 10 \\
\hline $11-6,16$ & 0.644 & 6.43 & 0.098 & 48 & 65 & 335 & 114 & 81 & 127 & 24 & 151 & 11 & 343 & 37 & 75 & 30 & 12 \\
\hline & 6 & & & 47 & 65 & 222 & 153 & 80 & 124 & 23 & 1 & 12 & 390 & 33 & 89 & 19 & 14 \\
\hline $12-2,33$ & 0.64 & 5.41 & & 8 & 8 & - & 183 & 102 & 13 & 34 & 17 & 15 & 455 & 56 & 120 & 25 & 16 \\
\hline $12-6,128$ & 0.991 & 7.67 & 0.113 & 73 & 54 & 276 & 116 & 108 & 138 & 30 & 172 & 13 & 373 & 52 & 117 & 20 & 19 \\
\hline
\end{tabular}

there is an equivalent sharp change in chemistry. The reverse situation is seen in Core 8 where there is a change within the core from micrite-diatom ooze to diatom-micrite ooze. But Core 9 sees a return to diatom-rich oozes.

Core 22 markes another abrupt change in chemistry which correlates with a change from diatom oozes to nanno chalks and coincides with the paleontological boundary between upper and middle Miocene. Below this the lithology is a more geochemically uniform nanno chalk, but there is a minor break at Core 27 which coincides with a hiatus between middle Miocene and Oligocene.

The reasons for these variations in geochemistry may be complex. Medium- to long-term variations may be related to fluctuations in the CCD, changes in the relative productivity and type of siliceous and calcareous organisms, or variations in the current activity or sediment source. Some of these shanges appear to have taken place gradually; others very rapidly.

\section{Site 328}

Downhole variations at Site 328 are shown in Figure 2 and displayed in a similar manner to those for Site 329 in Figure 1. However, there are complications in that, apart from the first three cores, Hole 328 was cored discontinuously with only moderate recovery and with appreciable gaps between each core. Hole 328B yielded six continuous cores (Cores 1-6) which overlap Cores 2-4 of Hole 328, but Core 7 of Hole 328B lay stratigraphically below Core 12 of Hole 328 . Hence in Figure 2, Core 7 of Hole 328B is included with the Hole 328 results.

Hole $328 \mathrm{~B}$ is plotted separately and was sampled primarily to assess the geochemical differences between the biogenous siliceous clays of Pliocene-Miocene age (Cores 2-4) and the underlying zeolitic clays of the Oligocene (Cores 5 and 6).

The relevant geochemical features of Site 328 can be summarized as follows: 
TABLE 2

Geochemical Data for Siliceous Clays, Zeolitic Clays, and Claystones,

Hole 328B, Malvinas Outer Basin (oxides in \%, trace elements in ppm)

\begin{tabular}{|c|c|c|c|c|c|c|c|c|c|c|c|c|c|c|c|c|c|}
\hline $\begin{array}{c}\text { Sample } \\
\text { (Interval } \\
\text { in } \mathrm{cm} \text { ) }\end{array}$ & $\mathrm{TiO}_{2}$ & $\mathrm{Fe}_{2} \mathrm{O}_{3}$ & $\mathrm{MnO}$ & $\mathrm{Cr}$ & $\mathrm{Ni}$ & $\mathrm{Cu}$ & $\mathrm{Zn}$ & $\mathrm{Rb}$ & $\mathrm{Sr}$ & $\mathrm{Y}$ & $\mathrm{Zr}$ & $\mathrm{Nb}$ & $\mathrm{Ba}$ & $\mathrm{La}$ & $\mathrm{Ce}$ & $\mathrm{Pb}$ & $\mathrm{Th}$ \\
\hline $2-5,121$ & - & - & - & 35 & 164 & - & 143 & 99 & 176 & 30 & 205 & 11 & 1388 & 39 & 65 & 17 & 13 \\
\hline $2-6,121$ & 0.958 & 8.64 & 0.375 & 38 & 147 & 533 & 193 & 103 & 173 & 31 & 206 & 12 & 1216 & 40 & 72 & 19 & 11 \\
\hline $3-1,97$ & - & - & - & 34 & 101 & 392 & 155 & 83 & 154 & 25 & 188 & 11 & 751 & 35 & 67 & 21 & 9 \\
\hline $3-2,95$ & - & - & - & 37 & 156 & - & - & - & - & - & 179 & 9 & - & 34 & 59 & - & - \\
\hline $3-3,91$ & 0.854 & 7.69 & 0.566 & 32 & 487 & 414 & 160 & 85 & 147 & 25 & 167 & 10 & 803 & 33 & 57 & 18 & 10 \\
\hline $3-4,95$ & 0.651 & 6.40 & 0.280 & 28 & 114 & 305 & 135 & 80 & 142 & 25 & 155 & 8 & 613 & 27 & 49 & 13 & 7 \\
\hline $3-5,121$ & - & - & - & 36 & 226 & 435 & 158 & 88 & 153 & 25 & 160 & 9 & 918 & 31 & 56 & 19 & 11 \\
\hline $4-1,118$ & 0.814 & 7.67 & 0.216 & 57 & 87 & - & 157 & 128 & 147 & 29 & 168 & 11 & 922 & 36 & 73 & 16 & 15 \\
\hline $4-2,111$ & 0.805 & 7.81 & 0.622 & 52 & 183 & 295 & 172 & 150 & 138 & 28 & 173 & 10 & 782 & 43 & 80 & 25 & 18 \\
\hline $4-3,104$ & 0.602 & 7.74 & 0.545 & 55 & 163 & 308 & 171 & 146 & 136 & 28 & 170 & 10 & 772 & 39 & 76 & 23 & 16 \\
\hline $4-4,121$ & 0.653 & 7.70 & 0.508 & 52 & 164 & 299 & 176 & 147 & 135 & 42 & 178 & 10 & 733 & 36 & 77 & 26 & 17 \\
\hline $4-5,135$ & - & - & - & 36 & 170 & 216 & 135 & 99 & 104 & 18 & 129 & $T$ & 432 & 21 & 50 & 20 & 11 \\
\hline $5-1,81$ & - & - & - & 52 & 97 & 248 & 204 & 116 & 114 & 35 & 172 & 11 & 364 & 56 & 150 & 17 & 17 \\
\hline $5-2,91$ & 0.954 & 8.78 & 0.806 & 51 & 186 & 283 & 205 & 113 & 153 & 30 & 187 & 11 & 733 & 51 & 116 & 21 & 15 \\
\hline $5-3,100$ & 0.646 & 7.08 & 0.523 & 43 & 121 & 224 & 196 & 99 & 128 & 27 & 170 & 10 & 347 & 45 & 152 & 15 & 17 \\
\hline $5-4,121$ & 0.976 & 8.98 & 0.362 & 48 & 113 & - & 192 & 103 & 141 & 27 & 177 & 11 & 435 & 41 & 94 & 19 & 14 \\
\hline $5-5,129$ & - & - & - & 51 & 126 & 256 & 192 & 112 & 131 & 27 & 168 & 10 & 585 & 44 & 113 & 26 & 13 \\
\hline $5-6,81$ & 0.924 & 9.09 & 0.910 & 52 & 215 & 263 & 216 & 111 & 132 & 25 & 166 & 11 & 603 & 40 & 99 & 30 & 14 \\
\hline $6-1,32$ & 1.031 & 10.02 & 0.304 & 63 & 117 & 250 & 172 & 122 & 104 & 35 & 153 & 10 & 426 & 56 & 141 & 34 & 19 \\
\hline $6-2,82$ & 0.885 & 9.41 & 0.256 & 62 & 108 & 244 & 17 & 119 & 97 & 31 & 15 & 11 & 343 & 46 & 123 & 28 & 20 \\
\hline $6-3,102$ & - & - & - & 63 & 114 & - & 180 & 116 & 107 & 33 & 157 & 11 & 330 & 51 & 122 & 29 & 17 \\
\hline $6-4,102$ & 1.025 & 9.75 & 0.298 & 69 & 119 & 260 & 195 & 117 & 106 & 33 & 159 & 12 & 423 & 50 & 117 & 22 & 18 \\
\hline $6-5,102$ & - & - & - & 66 & 114 & - & 175 & 117 & 104 & 33 & 161 & 14 & 377 & 54 & 118 & 21 & 18 \\
\hline $6-6,86$ & - & - & - & 71 & 131 & - & 117 & 122 & 108 & 32 & 152 & 13 & 457 & 51 & 122 & 30 & 18 \\
\hline $7-1,97$ & - & - & - & 70 & 176 & 293 & 11 & 94 & 149 & 25 & 17 & 15 & 452 & 45 & 123 & 19 & 13 \\
\hline $7-2,98$ & 0.489 & 11.10 & 0.154 & 57 & 68 & 203 & 103 & 99 & 131 & 21 & 163 & 14 & 307 & 46 & 92 & 20 & 15 \\
\hline $7-3,112$ & 0.868 & 5.37 & 0.133 & 70 & 90 & 232 & 143 & 90 & 149 & 22 & 180 & 15 & 419 & 40 & 100 & 21 & 16 \\
\hline $7-4,25$ & 0.841 & 14.19 & 0.178 & 49 & 81 & 183 & 103 & 96 & 133 & 24 & 148 & 12 & 253 & 48 & 112 & 25 & 12 \\
\hline & - & - & - & 66 & 96 & 221 & 13 & 92 & 141 & 24 & 18 & 14 & 205 & 48 & 112 & 26 & 18 \\
\hline $7-6,56$ & 0.094 & 10.50 & 0.253 & 60 & 78 & 222 & 118 & 103 & 142 & 50 & 178 & 17 & 679 & 76 & 227 & 27 & 15 \\
\hline
\end{tabular}

1) Although there is some geochemical coherence in the downhole element variations, the strong sympathetic and antipathetic relationships noted for Site 329 are much less well developed. The most marked change relates to $\mathrm{Sr}$, which had a strong antipathetic relationship with $\mathrm{Zn}, \mathrm{Rb}, \mathrm{Cr}, \mathrm{Ni}, \mathrm{Th}, \mathrm{Pb}$, and most other elements at Site 329, but here varies sympathetically with $\mathrm{Zn}, \mathrm{Rb}, \mathrm{Ba}$, and $\mathrm{Zn}$, at least with respect to short- and medium-term variations. Likewise, $\mathrm{Ni}$ and $\mathrm{Cr}$, while varying sympathetically with each other and with $\mathrm{Zn}, \mathrm{Rb}, \mathrm{Th}$, and $\mathrm{Pb}$ in Hole 329 , now display very little geochemical coherence with these elements, nor indeed with each other, expecially in Hole 328B.

2) Long- and medium-term trends are displayed by many elements. La and Ce show an increasing concentration with depth in both Holes 328 and 328B, while Y shows little long-term variation. The significance of this is difficult to understand, but implies that rare-earth element patterns might be increasingly light-RE enriched with depth in the core. $\mathrm{Cr}$ shows a similar increase in Hole 328B. On the other hand, $\mathrm{Zn}$ increases between Cores 1-4 in Hole 328, and then decreases for the remainder of the core. Similar variations are apparent for other trace elements, but there is no consistent pattern of long-term behavior. This would suggest that there is no single variable responsible for these trends, but that each element is acting independently. The most likely explanation is that the variations reflect a constantly changing source of sediment. Short-term variations, where several elements show simultaneous fluctuations in abundance (although to variable degrees) may be linked to new sediment sources or changing bottom currents.

3) Referring to the continuously cored section in Hole 328B which records the lithological change between the Pliocene-Miocene siliceous clays (Cores 2, 3 , and 4) and the zeolitic clays of Cores 5 and 6, there are certainly sharp fluctuations for many elements at the boundary between Core 4 and Core 5 . This is most marked for $\mathrm{Zn}, \mathrm{Cr}, \mathrm{La}$, and $\mathrm{Ce}$ which are of lower abundance in the siliceous clays, while $\mathrm{Rb}, \mathrm{Sr}$, and $\mathrm{Ba}$ are more concentrated in the siliceous clays. On the other hand, there is no obvious change in $\mathrm{Ni}, \mathrm{Y}, \mathrm{Th}$, and $\mathrm{Pb}$. However, with $\mathrm{Ba}, \mathrm{Cr}, \mathrm{Ce}$, and $\mathrm{La}$, the changes are merely an accentuation of longer term trends evident in this core, and a sharper discontinuity might have been expected if the Core 4 /Core 5 boundary also represents a hiatus in sedimentation.

The equivalent horizon in Hole 328 lies between Cores 3 and 4 . In general, the same geochemical differences are evident in that $\mathrm{Zn}, \mathrm{Cr}, \mathrm{La}$, and $\mathrm{Ce}$ have lower concentrations in Cores 1 and 3 than in the deeper parts of the core, while $\mathrm{Zr}$ and $\mathrm{Sr}$ have higher values. $\mathrm{Ni}$ is distinctly higher in Cores 3 to 5 than in the sediments above and below. This interval obviously coincides with Cores 2 to 6 in Hole 328B. The high Ni content is clearly related to the high proportion of manganese micronodules at this point in the section.

In summary, the geochemical variations in the sediments at Site 328 are more complex than at Site 329. While some of these variations might be connected 
TABLE 3

Geochemical Data for Siliceous-Calcareous Sediments at Site 329, Falkland Plateau (oxices in \%, trace elements in ppm)

\begin{tabular}{|c|c|c|c|c|c|c|c|c|c|c|c|c|c|c|c|c|c|}
\hline $\begin{array}{c}\text { Sample } \\
\text { (Interval in } \mathrm{cm} \text { ) }\end{array}$ & $\mathrm{TiO}_{2}$ & $\mathrm{Fe}_{2} \mathrm{O}_{3}$ & Mn0 & $\mathrm{Cr}$ & $\mathrm{Ni}$ & $\mathrm{Cu}$ & $\mathrm{Zn}$ & $\mathrm{Rb}$ & $\mathrm{Sr}$ & $\mathrm{Y}$ & $\mathrm{Zr}$ & $\mathrm{Nb}$ & $\mathrm{Ba}$ & $\mathrm{La}$ & $\mathrm{Ca}$ & $\mathrm{Pb}$ & Th \\
\hline $1-1,131$ & 0.263 & 7.36 & 0.099 & 103 & 25 & 168 & 124 & 98 & 140 & 15 & 200 & 15 & 484 & 15 & 21 & 10 & 5 \\
\hline $1-2,81$ & 0.351 & 6.91 & 0.103 & 87 & 34 & 166 & 135 & 87 & 124 & 17 & 192 & 16 & 575 & 20 & 27 & 16 & 10 \\
\hline $1-1,41$ & 0.218 & 6.47 & 0.100 & 59 & 33 & 183 & 137 & 79 & 111 & 23 & 130 & 6 & 548 & 14 & 15 & 13 & 1 \\
\hline $1-4,41$ & 0.129 & 2.11 & 0.094 & 12 & 19 & 168 & 59 & 28 & 625 & 15 & 80 & 5 & 583 & 9 & 12 & 6 & 5 \\
\hline $1-5,131$ & 0.113 & 2.83 & 0.096 & 8 & 21 & 193 & 58 & 22 & 648 & 10 & 76 & 4 & 531 & 8 & 8 & 7 & 6 \\
\hline $1-6,49$ & 0.250 & 2.68 & 0.104 & 9 & 18 & 193 & 68 & 27 & 567 & 12 & 99 & 4 & 490 & 11 & 20 & 9 & 3 \\
\hline $2-1,121$ & 0.318 & 4.54 & 0.104 & 1190 & 282 & 180 & 139 & 55 & 463 & 28 & 97 & 5 & 936 & 9 & 1 & 9 & 3 \\
\hline $2-2,102$ & 0.340 & 5.08 & 0.106 & 39 & 38 & 195 & 132 & 56 & 418 & 29 & 128 & 2 & 944 & 20 & 23 & 6 & 3 \\
\hline $2-3,131$ & 0.128 & 2.21 & 0.098 & 8 & 17 & 172 & 58 & 29 & 866 & 13 & 100 & 4 & 306 & 7 & 11 & 5 & 6 \\
\hline $2-4,132$ & 0.109 & 2.75 & 0.093 & 8 & 14 & 167 & 45 & 22 & 1087 & 13 & 87 & 3 & 268 & 8 & 12 & 10 & 1 \\
\hline $2-5,83$ & 0.120 & 2.07 & 0.100 & 8 & 20 & 174 & 55 & 38 & 922 & 15 & 94 & 4 & 358 & 8 & 9 & 3 & 2 \\
\hline $2-6,71$ & 0.098 & 1.87 & 0.099 & 8 & 15 & 185 & 51 & 23 & 919 & 11 & 83 & 5 & 326 & 7 & 11 & 9 & 2 \\
\hline $3-1,67$ & 0.158 & 2.50 & 0.097 & 18 & 22 & 174 & 74 & 35 & 597 & 14 & 95 & 4 & 480 & 9 & 14 & 8 & 5 \\
\hline $3-2,82$ & 0.179 & 2.36 & 0.094 & 10 & 16 & 168 & 59 & 29 & 865 & 11 & 98 & 4 & 339 & 8 & 11 & 7 & 2 \\
\hline $3-3,72$ & 0.204 & 3.39 & 0.099 & 13 & 24 & 169 & 51 & 26 & 749 & 7 & 97 & 3 & 316 & 10 & 11 & 7 & 3 \\
\hline $3-4,89$ & 0.186 & 2.37 & 0.100 & 14 & 22 & 191 & 71 & 30 & 786 & 12 & 100 & 4 & 364 & 10 & 13 & 8 & 1 \\
\hline $3-6,116$ & 0.072 & 2.40 & 0.095 & 4 & 6 & 163 & 30 & 12 & 1242 & 6 & 73 & 2 & 275 & 5 & 7 & 3 & 1 \\
\hline $4-1,108$ & 0.168 & 2.34 & 0.096 & 11 & 21 & 193 & 70 & 28 & 572 & 11 & 91 & 4 & 557 & 8 & 12 & 4 & 1 \\
\hline $4-3,105$ & 0.260 & 2.83 & 0.098 & 22 & 45 & 253 & 120 & 37 & 181 & 15 & 94 & 4 & 794 & 13 & 18 & 8 & 7 \\
\hline $4-5,26$ & 0.180 & 1.57 & 0.097 & 11 & 25 & 211 & 89 & 35 & 257 & 15 & 95 & 4 & 455 & 6 & 9 & 9 & 6 \\
\hline $5-1,62$ & 0.160 & 1.84 & 0.101 & 14 & 30 & 222 & 95 & 35 & 342 & 14 & 95 & 6 & 676 & 8 & 14 & 6 & 3 \\
\hline $5-4,76$ & 0.252 & 2.46 & 0.103 & 20 & 30 & 225 & 112 & 36 & 306 & 16 & 98 & 4 & 814 & 14 & 14 & 5 & 3 \\
\hline $5-6,123$ & 0.100 & 1.72 & 0.093 & 14 & 23 & 207 & 97 & 35 & 237 & 15 & 92 & 5 & 423 & 5 & 10 & 7 & 3 \\
\hline $6-2,126$ & 0.151 & 1.44 & 0.098 & 12 & 22 & 205 & 79 & 26 & 427 & 12 & 79 & 3 & 528 & 10 & 11 & 5 & 1 \\
\hline $6-5,84$ & 0.076 & 1.50 & 0.094 & 9 & 27 & 218 & 88 & 34 & 314 & 13 & 90 & 3 & 261 & 8 & 7 & 11 & 5 \\
\hline $7-6,86$ & 0.115 & 1.06 & 0.095 & 8 & 25 & 218 & 68 & 22 & 503 & 9 & 71 & 4 & 510 & 7 & 11 & 8 & 3 \\
\hline $8-2,125$ & 0.074 & 1.58 & 0.093 & 5 & 15 & 170 & 45 & 15 & 879 & 9 & 70 & 2 & 258 & 5 & 6 & 6 & 2 \\
\hline $8-5,56$ & 0.043 & 2.23 & 0.093 & 3 & 6 & 154 & 26 & 8 & 1234 & 5 & 62 & 1 & 134 & 4 & 10 & 2 & 1 \\
\hline $9-4,137$ & 0.178 & 2.74 & 0.101 & 13 & 29 & 187 & 87 & 41 & 562 & 18 & 112 & 3 & 459 & 9 & 13 & 8 & 4 \\
\hline $9-5,86$ & 0.242 & 2.99 & 0.100 & 18 & 29 & 193 & 93 & 44 & 592 & 18 & 114 & 5 & 631 & 11 & 16 & 10 & 8 \\
\hline $10-1,114$ & 0.138 & 2.18 & 0.097 & 10 & 20 & 185 & 76 & 30 & 593 & 12 & 88 & 5 & 541 & 6 & 13 & 9 & 5 \\
\hline $10-4,58$ & 0.065 & 1.69 & 0.091 & 6 & 18 & 187 & 54 & 20 & 595 & 8 & 67 & 1 & 332 & 4 & 4 & 4 & 1 \\
\hline $10-6,60$ & 0.067 & 1.59 & 0.093 & 10 & 15 & 185 & 46 & 16 & 901 & 7 & 67 & 1 & 275 & 4 & 9 & 4 & 1 \\
\hline $11-2,66$ & 0.090 & 1.67 & 0.090 & 6 & 15 & 185 & 50 & 15 & 924 & 6 & 72 & 4 & 362 & 4 & 7 & 3 & 1 \\
\hline $11-5,19$ & 0.115 & 1.87 & 0.093 & 11 & 17 & 183 & 57 & 21 & 810 & 9 & 77 & 2 & 428 & 6 & 10 & 5 & 2 \\
\hline $12-2,26$ & 0.118 & 1.99 & 0.097 & 9 & 18 & 174 & 66 & 21 & 757 & 9 & 77 & 3 & 467 & 8 & 10 & 8 & 1 \\
\hline $12-5,112$ & 0.147 & 2.05 & 0.097 & 18 & 21 & 189 & 63 & 27 & 838 & 10 & 85 & 2 & 627 & 8 & 8 & 4 & 3 \\
\hline $13-2,23$ & 0.184 & 2.10 & 0.101 & 15 & 32 & 225 & 112 & 43 & 359 & 13 & 109 & 5 & 483 & 9 & 12 & 12 & 4 \\
\hline $13-4 ; 41$ & 0.126 & 2.10 & 0.096 & 9 & 24 & 185 & 62 & 26 & 732 & 12 & 83 & 2 & 475 & 6 & 12 & 7 & 2 \\
\hline $14-2,69$ & 0.084 & 1.66 & 0.097 & 7 & 11 & 187 & 46 & 20 & 899 & 14 & 79 & 2 & 370 & 7 & 8 & 6 & 1 \\
\hline $14-6,62$ & 0.086 & 1.71 & 0.096 & 8 & 16 & 176 & 49 & 21 & 1089 & 10 & 82 & 3 & 420 & 6 & 9 & 5 & 2 \\
\hline $15-2,62$ & - & $\overline{-}$ & - & 6 & 16 & 152 & 29 & 14 & 1224 & 8 & 79 & 2 & 171 & 7 & 9 & 4 & 2 \\
\hline $15-3,139$ & 0.087 & 2.59 & 0.097 & 7 & 15 & 156 & 34 & 16 & 1202 & 7 & 76 & 2 & 237 & 6 & 7 & 7 & 1 \\
\hline $15-5,123$ & 0.100 & 1.75 & 0.098 & 9 & 15 & 176 & 39 & 21 & 1142 & 8 & 84 & 2 & 324 & 7 & 16 & 5 & 1 \\
\hline $16-1,80$ & 0.127 & 1.99 & 0.099 & 9 & 20 & 185 & 53 & 23 & 932 & 10 & 85 & 4 & 419 & 9 & 11 & 5 & 1 \\
\hline $16-4,63$ & 0.037 & 2.21 & 0.094 & 1 & 6 & 163 & 21 & 9 & 1348 & 6 & 66 & 2 & 130 & 5 & 6 & 6 & 1 \\
\hline $17-2,88$ & 0.062 & 1.43 & 0.094 & 4 & 11 & 180 & 36 & 11 & 1068 & 6 & 67 & 2 & 281 & 6 & 11 & 5 & 1 \\
\hline $17-4,54$ & 0.050 & 2.29 & 0.093 & 6 & 6 & 158 & 28 & 10 & 1149 & 7 & 63 & 1 & 193 & 4 & 6 & 4 & 3 \\
\hline $18-4,129$ & 0.086 & 2.59 & 0.098 & 5 & 11 & 159 & 38 & 20 & 1147 & 9 & 82 & 2 & 255 & 5 & 9 & 6 & 2 \\
\hline $18-6,26$ & 0.130 & 1.93 & 0.094 & 11 & 19 & 187 & 58 & 22 & 853 & 10 & 83 & 2 & 399 & 5 & 10 & 6 & 3 \\
\hline $19-2,123$ & 0.080 & 1.63 & 0.095 & 7 & 16 & 174 & 43 & 15 & 886 & 7 & 70 & 3 & 206 & 6 & 7 & 7 & 1 \\
\hline $19-3,105$ & 0.139 & 2.15 & 0.098 & 10 & 19 & 180 & 62 & 27 & 602 & 10 & 80 & 5 & 312 & 6 & 10 & 11 & 4 \\
\hline $20-2,39$ & 0.106 & 1.14 & 0.091 & 6 & 22 & 192 & 57 & 21 & 444 & 8 & 69 & 4 & 368 & 5 & 9 & 7 & 1 \\
\hline $20-5,113$ & 0.082 & 1.59 & 0.097 & 7 & 13 & 180 & 42 & 16 & 729 & 9 & 66 & 3 & 357 & 5 & 9 & 6 & 3 \\
\hline $21-3,31$ & 0.124 & 1.81 & 0.097 & 12 & 28 & 214 & 92 & 32 & 254 & 12 & 100 & 4 & 465 & 9 & 9 & 7 & 3 \\
\hline $21-4,59$ & 0.107 & 1.91 & 0.099 & 10 & 18 & 180 & 54 & 24 & 854 & 14 & 86 & 2 & 522 & 8 & 11 & 4 & 3 \\
\hline $22-2,47$ & 0.086 & 1.17 & 0.094 & 8 & 24 & 201 & 71 & 23 & 179 & 6 & 67 & 3 & 366 & 6 & 5 & 5 & 3 \\
\hline $22-4,109$ & 0.103 & 1.74 & 0.096 & 9 & 12 & 191 & 46 & 17 & 818 & 11 & 74 & 1 & 628 & 7 & 10 & 4 & 1 \\
\hline $23-1,98$ & 0.171 & 2.24 & 0.091 & 12 & 23 & 197 & 62 & 29 & 805 & 9 & 90 & 2 & 501 & 8 & 11 & 8 & 3 \\
\hline $23-2,44$ & 0.087 & 1.58 & 0.091 & 6 & 13 & 183 & 42 & 17 & 1259 & 10 & 81 & 1 & 298 & 8 & 13 & 3 & 5 \\
\hline $23-3,132$ & 0.076 & 2.45 & 0.092 & 6 & 11 & 160 & 32 & 15 & 1406 & 9 & 77 & 1 & 333 & 4 & 9 & 5 & 2 \\
\hline $14-2,132$ & 0.133 & 1.96 & 0.095 & 9 & 16 & 183 & 54 & 24 & 926 & 10 & 84 & 2 & 471 & 8 & 7 & 4 & 4 \\
\hline $24-3,123$ & 0.084 & 1.65 & 0.094 & 10 & 13 & 170 & 46 & 24 & 970 & 15 & 82 & 2 & 359 & 8 & 6 & 8 & 5 \\
\hline $26-1,65$ & 0.084 & 1.63 & 0.093 & 10 & 15 & 178 & 43 & 18 & 943 & 9 & 75 & 1 & 393 & 7 & 6 & 5 & 4 \\
\hline $26-3,106$ & 0.192 & 1.82 & 0.09 & 14 & 25 & 218 & 74 & 21 & 143 & 5 & 64 & 3 & 40 & 9 & 8 & 6 & 4 \\
\hline $27-1,31$ & 0.103 & 2.57 & 0.09 & 5 & 10 & 152 & 33 & 16 & 1205 & 14 & 77 & 1 & 997 & 8 & 12 & 8 & 2 \\
\hline $28-1,132$ & 0.113 & 2.88 & 0.112 & 11 & 26 & 154 & 43 & 29 & 1120 & 11 & 82 & 3 & 458 & 8 & 13 & 5 & 2 \\
\hline
\end{tabular}


TABLE 4

Average Element Compositions for Site 328 Siliceous and Zeolitic Clays and Site 329

Siliceous and Calcareous Oozes, with Standard Deviations

and Maximum and Minimum Values for Each Element

\begin{tabular}{|c|c|c|c|c|c|c|c|c|c|}
\hline & \multirow[b]{2}{*}{ Mean } & \multicolumn{2}{|c|}{ Site 328} & \multicolumn{6}{|c|}{ Site 329} \\
\hline & & S. D. & Max. & Min & Mean & S. D. & Max & Min & $328 / 329$ \\
\hline $\mathrm{TiO}_{2}(\%)$ & 0.734 & 0.176 & 1.031 & 0.209 & 0.134 & 0.067 & 0.351 & 0.037 & 5.5 \\
\hline $\mathrm{Fe}_{2} \mathrm{O}_{3}(\%)$ & 7.76 & 1.99 & 14,19 & 3.79 & 2.40 & 1.16 & 7.36 & 1,06 & 3.2 \\
\hline $\operatorname{Mn} 0(\%)$ & 0.272 & 0.297 & 1.790 & 0.094 & 0.098 & 0.005 & 0.112 & 0.090 & 2.8 \\
\hline $\mathrm{Cr}^{\mathrm{a}}$ & 82 & 146 & 1089 & 28 & 29 & 139 & 1190 & $<1$ & 2.8 \\
\hline $\mathrm{Ni}$ & 117 & 80 & 487 & 40 & 23 & 32 & 282 & 6 & 5.1 \\
\hline $\mathrm{Cu}$ & 293 & - & 593 & 164 & 182 & 21 & 253 & 150 & 1.6 \\
\hline $\mathrm{Zn}$ & 150 & 32 & 216 & 96 & 62 & 29 & 139 & 21 & 2.4 \\
\hline $\mathrm{Rb}$ & 100 & 17 & 150 & 64 & 27 & 16 & 98 & 8 & 3.7 \\
\hline $\mathrm{Sr}$ & 135 & 18 & 176 & 97 & 757 & 335 & 1406 & 111 & 0.2 \\
\hline $\mathrm{Y}$ & 26 & 6 & 50 & 15 & 12 & 5 & 29 & 5 & 2.2 \\
\hline $\mathrm{Zr}$ & 163 & 41 & 406 & 117 & 86 & 24 & 200 & 49 & 1.9 \\
\hline $\mathrm{Nb}$ & 12 & 3 & 32 & 6 & 3 & 2.5 & 16 & $<1$ & 4.0 \\
\hline $\mathrm{Ba}$ & 558 & - & 1367 & 291 & 463 & 194 & 997 & 130 & 1.2 \\
\hline $\mathrm{La}$ & 39 & 10 & 76 & 17 & 8 & 4 & 20 & 4 & 4.9 \\
\hline $\mathrm{Ce}$ & 84 & 35 & 227 & 30 & 12 & 5 & 28 & $<1$ & 7.0 \\
\hline $\mathrm{Pb}$ & 20 & 7 & 34 & 10 & 7 & 3 & 16 & 2 & 2.9 \\
\hline Th & 14 & 4 & 20 & 5 & 3 & 2 & 10 & $<1$ & 4.7 \\
\hline $\mathrm{Cr} / \mathrm{Ni}$ & 0.70 & & 4.09 & 0.07 & 1.23 & & 4.2 & 0.10 & 0.6 \\
\hline $\mathrm{Fe} / \mathrm{Mn}$ & 16.5 & & 98.2 & 3.1 & 24.5 & & 74 & 11.1 & 0.7 \\
\hline $\mathrm{Rb} / \mathrm{Sr}$ & 0.74 & & 1.23 & 0.47 & 0.036 & & 0.71 & 0.006 & 20.6 \\
\hline $\mathrm{Ba} / \mathrm{Rb}$ & 5.4 & & 14.02 & 2.64 & 17.1 & & 62 & 4.9 & 0.3 \\
\hline $\mathrm{Ba} / \mathrm{Sr}$ & 4.0 & & 7.89 & 1.48 & 0.61 & & 4.9 & 0.10 & 6.6 \\
\hline
\end{tabular}

with the increase in biogenous components in the upper part of the core, or with the varying adsorption of cations by the clay and zeolite components, or with the presence of manganese nodules, the general lack of geochemical coherence between the trace elements suggests that the main variable might be the source of the sediments.

\section{GEOCHEMICAL COMPARISONS BETWEEN SITES 328 AND 329}

Table 4 shows a comparison of mean values of Site 328 and Site 329 sediments and illustrates the distinctly higher trace-element values ( $\mathrm{Sr}$ excepted) in a deep water site compared with a shallow water site, a feature which has been noted previously (Cook, 1974). Mere comparison of averages can, however, obscure other geochemical relationships such as those noted above with regard to downhole variations in sediment chemistry.

In order to extend this study, correlation matrices, containing correlation coefficients between each different pair of elements, and some important element ratios, have been calculated using a computer program (CORRCLUST) written by A.C. Skinner. Combined correlation matrices for Site 328 and Site 329 sediments are tabulated in Figure 5. In order to clarify presentation, the coefficients have been increased by a factor of 100 (i.e., $R \times 100$ ) and quoted to only two significant figures. Correlation matrices have been used previously in the study of Indian Ocean sediments by Cook (1974), although his sample populations were rather small.

The correlation coefficient between two variables, $x$ and $y$, in a set of data is defined as the ratio of the covariance of the variables to the product of their standard deviations. Thus the correlation coefficient $(R)$ is given by

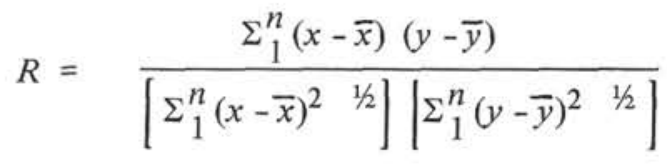

where $x$ and $y$ denote the values of the two variables, $\bar{x}$ and $\bar{y}$ are the means of the two variables in the set, and $n$ is the number of samples in the set.

However, it is also necessary to determine whether the correlation coefficients are significant or not since the significant values of the coefficients are dependent on the number of samples in the set, although this is of progressively less importance as the number of samples increases. Accordingly, Student's " $t$ " test has been used to calculate the significance of the correlation coefficients. In Table 5 those correlations which are significant at the $99.5 \%$ confidence level are shown in bold type. The limiting correlation coefficient value at this level of significance is \pm 0.27 for the sample size used. Both positive and negative correlations are shown.

The correlation matrices in Table 5 emphasize the geochemical differences between the two sites studied. It is not possible to examine or even explain all the interelement relationships, but certain features are worthy of particular note.

1) Some element pairs are strongly correlated at both sites, as may be expected from their normal geochemical coherence in many crustal rocks. Examples are: $\mathrm{Ce}-\mathrm{La}, \mathrm{Ce}-\mathrm{Y}$, La-Y, $\mathrm{Zr}-\mathrm{Nb}$, Th-Pb, Ti-Fe, $\mathrm{Cu}-\mathrm{Zn}, \mathrm{Fe}-\mathrm{La}, \mathrm{Fe}-\mathrm{Ce}$, Ti-La, Ti-Ce, Th-La, and Th-Ce.

2) At Site 329 there is a much higher level of correlation between most element pairs than at Site 328 . Furthermore, most of the significant correlation coefficients are positive. The main exception is $\mathrm{Sr}$, where all significant correlations are negative. An obvious reason 


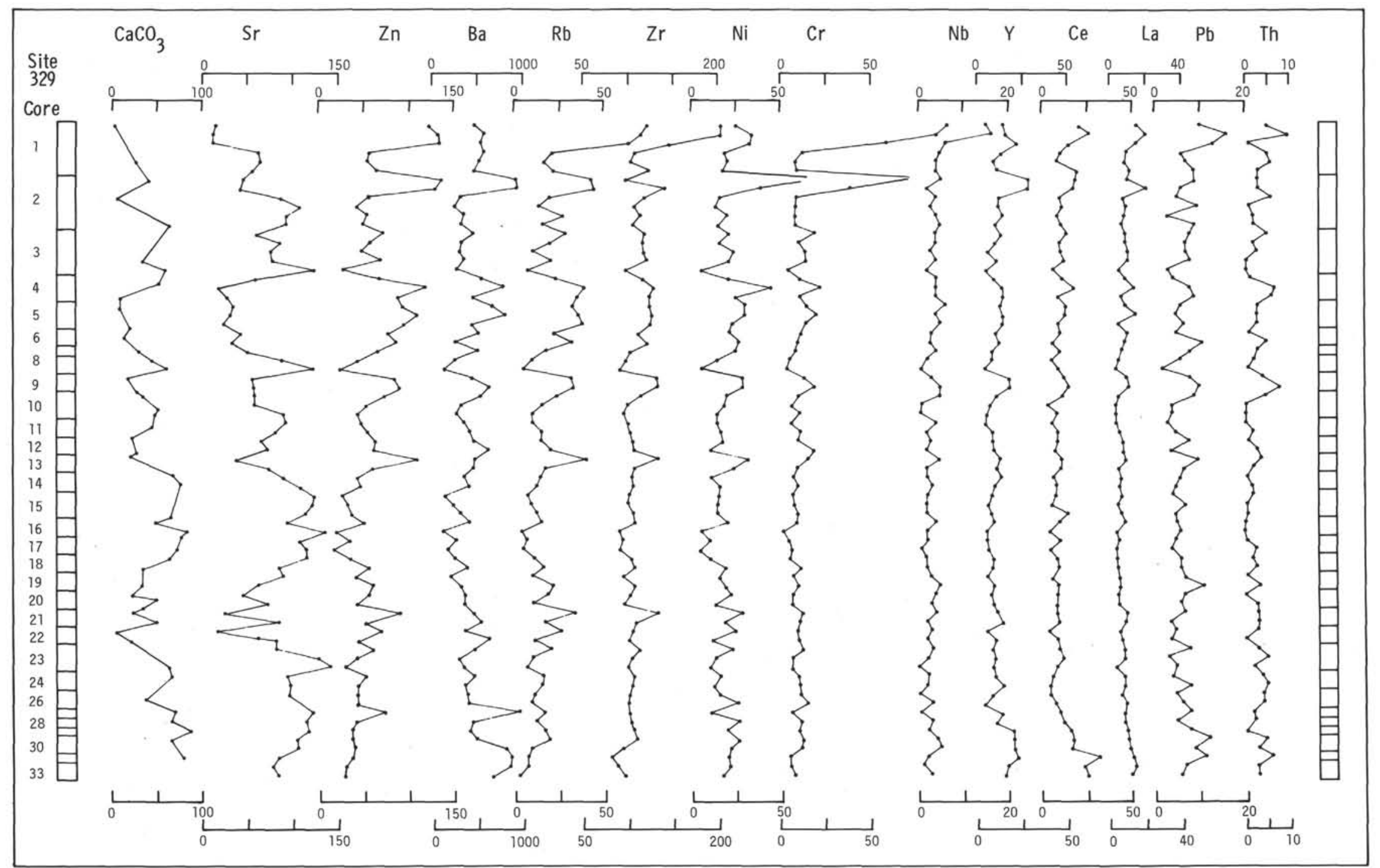

Figure 1. Downhole trace element variations in the diatom and nanno oozes and chalks at Site 329, Falkland Plateau. Cores $1-19$ are continuous to a depth of 180 meters, with intermittent coring to 464 meters in Cores 20-33. Vertical scale is not linear: samples are in incremental downhole order. 


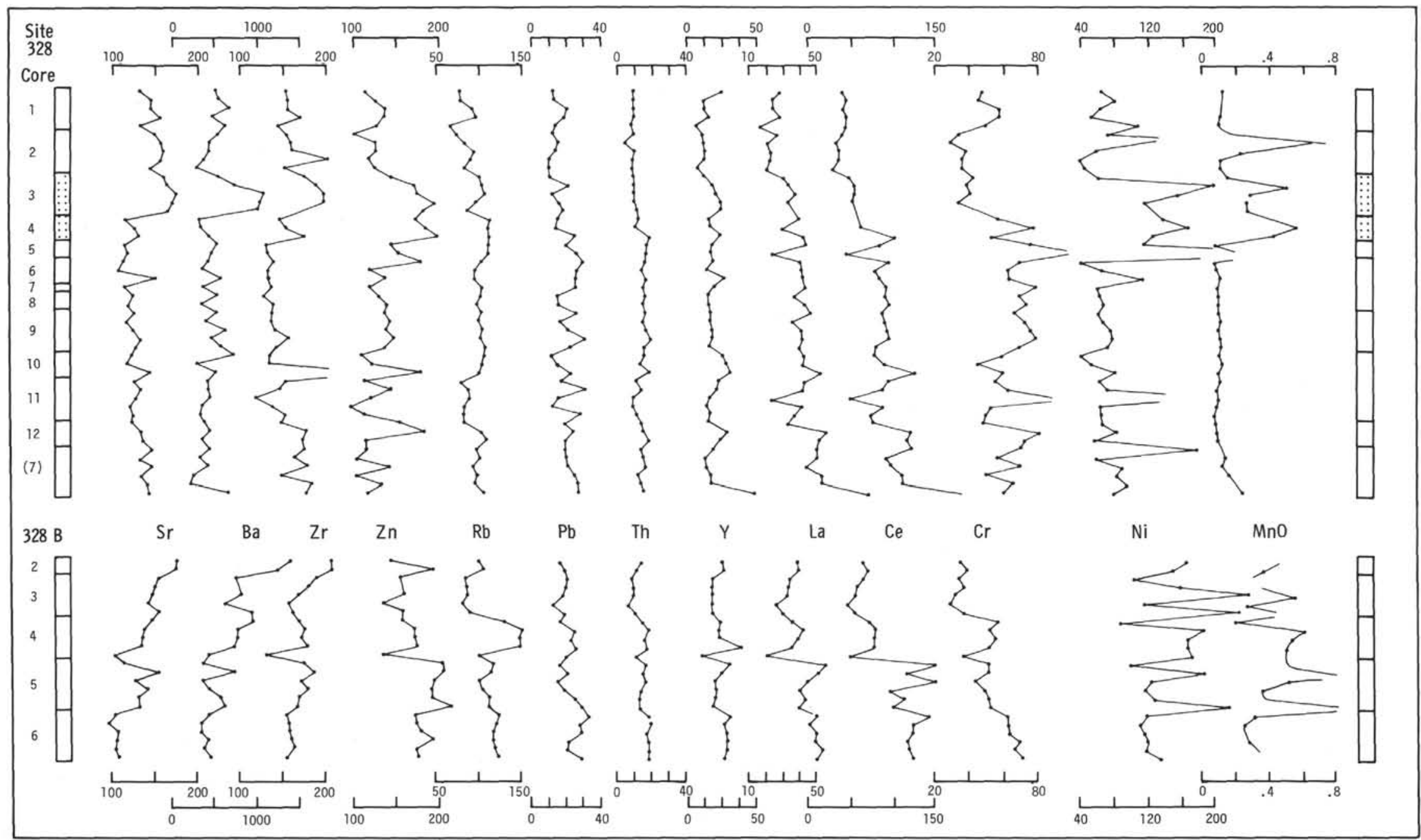

Figure 2. Downhole trace element variations in the siliceous and zeolitic clays and claystones at Site 328, Falkland Outer Basin. The upper part of the diagram covers the discontinuous coring in Hole 328 (and Hole 328B, Core 7) to a depth of 450 meters. The lower section represents the continuous coring, for 65 meters, in Hole 328B, through the transition between siliceous and zeolitic clays. This section is equivalent to the interval between (and including) Cores 3 and 4 in the upper diagram. Vertical scales are nonlinear: samples are in incremental downhole order. 
TABLE 5

Correlation Coefficients $(R \times 100)$ for Element Pairs in Deep-Sea Sediments at Site 328, Malvinas Outer Basin (Bottom Left) and Site 329, Falkland Plateau (top right)

\begin{tabular}{|c|c|c|c|c|c|c|c|c|c|c|c|c|c|c|c|c|c|c|c|c|c|c|c|}
\hline & $\mathrm{Ti}$ & $\mathrm{Fe}$ & $\mathrm{Mn}$ & $\mathrm{Cr}$ & $\mathrm{Ni}$ & $\mathrm{Cu}$ & $\mathrm{Zn}$ & $\mathrm{Rb}$ & Sr & $\mathrm{Y}$ & $\mathrm{Zr}$ & $\mathrm{Nb}$ & $\mathrm{Ba}$ & $\mathrm{La}$ & $\mathrm{Ce}$ & $\mathrm{Pb}$ & Th & $\mathrm{Cr} / \mathrm{Ni}$ & $\mathrm{Fe} / \mathrm{Mn}$ & $\mathrm{Rb} / \mathrm{Sr}$ & $\mathrm{Ba} / \mathrm{Rb}$ & $\mathrm{Ba} / \mathrm{Sr}$ & \\
\hline $\mathrm{Ti}$ & & 70 & 45 & 40 & 49 & 31 & 81 & 78 & -60 & 59 & 75 & 61 & 57 & 77 & 52 & 48 & 44 & 59 & 68 & 59 & 21 & 70 & \\
\hline $\mathrm{Ve}$ & 54 & & 44 & 32 & 31 & -23 & 55 & 82 & -29 & 56 & 80 & 67 & 36 & 72 & 55 & 53 & 32 & 75 & 99 & 80 & -16 & 64 & \\
\hline$M n$ & 1 & -5 & & 17 & 24 & -17 & 19 & 30 & -13 & 64 & 25 & 24 & 60 & 68 & 66 & 37 & 24 & 16 & 35 & 16 & 32 & 27 & $\hat{q}$ \\
\hline $\mathrm{Cr}$ & -39 & -26 & -12 & & 98 & 0 & 39 & 31 & -16 & 42 & 15 & 17 & 32 & 10 & -19 & 15 & 4 & 71 & 31 & 15 & -6 & 21 & N \\
\hline $\mathrm{Ni}$ & 1 & -13 & 67 & 25 & & 13 & 49 & 36 & -28 & 49 & 18 & 19 & 40 & 18 & -14 & 21 & 12 & 65 & 29 & 16 & -8 & 29 & o \\
\hline $\mathrm{Cu}$ & -13 & -47 & 8 & -19 & 8 & & 60 & 19 & -70 & -4 & 10 & 8 & 10 & 6 & -9 & 5 & 21 & -6 & -19 & 14 & -24 & 45 & 色 \\
\hline $\mathrm{Zn}$ & 47 & 0 & 41 & -13 & 38 & 17 & & 85 & -85 & 54 & 73 & 63 & 43 & 56 & 26 & 48 & 43 & 58 & 55 & 71 & -39 & 83 & ల \\
\hline $\mathrm{Rb}$ & 40 & 38 & 24 & -7 & 12 & -18 & 51 & & -65 & 58 & 93 & 82 & 35 & 66 & 43 & 59 & 45 & 74 & 82 & 88 & -42 & 77 & 8 \\
\hline $\mathrm{Sr}$ & -17 & -41 & 28 & -11 & 26 & 66 & 13 & -14 & & -31 & -49 & -56 & -35 & -44 & -20 & -43 & -43 & -34 & -29 & -62 & 25 & -79 & हु \\
\hline $\mathrm{Y}$ & 45 & 36 & 2 & -7 & 2 & -20 & 29 & 49 & -8 & & 47 & 29 & 71 & 70 & 54 & 50 & 35 & 41 & 51 & 35 & 10 & 45 & च \\
\hline $\mathrm{Zr}_{\mathrm{r}}$ & 15 & -17 & 10 & -18 & 7 & 27 & 37 & 13 & 42 & 31 & & 84 & 23 & 63 & 49 & 55 & 46 & 67 & 81 & 82 & -45 & 63 & స్ \\
\hline $\mathrm{Nb}$ & 19 & 2 & -17 & -6 & -15 & 0 & 9 & -1 & 12 & 32 & 80 & & 17 & 51 & 43 & 59 & 49 & 64 & 67 & 80 & -37 & 64 & $\tilde{z}$ \\
\hline $\mathrm{Ba}$ & 22 & -8 & 25 & -8 & 40 & 44 & 46 & 26 & 60 & 30 & 27 & -4 & & 66 & 51 & 26 & 32 & 26 & 30 & 19 & 56 & 47 & త్ర \\
\hline $\mathrm{La}$ & 60 & 65 & -12 & -17 & -18 & -39 & 14 & 42 & -32 & 70 & 18 & 42 & -1 & & 79 & 51 & 44 & 39 & 67 & 57 & 12 & 65 & 衣 \\
\hline $\mathrm{Ce}$ & 54 & 57 & -9 & -10 & -17 & -49 & 18 & 32 & -41 & 66 & 14 & 39 & -13 & 92 & & 47 & 41 & 12 & 50 & 40 & 26 & 42 & os \\
\hline $\mathrm{Pb}$ & 47 & 34 & 1 & -7 & 1 & -23 & 28 & 41 & -37 & 39 & 1 & 22 & -9 & 58 & 53 & & 49 & 34 & 51 & 54 & -15 & 47 & ते \\
\hline Th & 56 & 44 & -21 & -8 & -22 & -46 & 32 & 61 & -51 & 48 & 11 & 32 & -13 & 70 & 69 & 65 & & 22 & 30 & 36 & -7 & 44 & लn \\
\hline $\mathrm{Cr} / \mathrm{Ni}$ & -34 & -14 & -46 & 82 & -20 & -29 & -41 & -17 & -31 & -13 & -28 & 2 & -33 & -5 & -2 & -5 & 7 & & 76 & 68 & -25 & 52 & $\stackrel{\mathscr{E}}{\pi}$ \\
\hline $\mathrm{Fe} / \mathrm{Mn}$ & -1 & 38 & -68 & -1 & -64 & -35 & -60 & -25 & -48 & -13 & -28 & 15 & -44 & 24 & 17 & 9 & 26 & 47 & & 82 & -20 & 63 & \\
\hline $\mathrm{Rb} / \mathrm{Sr}$ & 42 & 51 & 3 & -1 & -4 & -46 & 33 & 81 & -67 & 42 & -14 & -8 & -14 & 49 & 46 & 51 & 73 & 2 & 5 & & -33 & 86 & \\
\hline $\mathrm{Ba} / \mathrm{Rb}$ & 6 & -26 & 14 & -6 & 37 & 52 & 24 & -20 & 62 & 7 & 20 & -6 & 89 & -23 & -28 & -27 & -40 & -28 & -36 & -49 & & -13 & \\
\hline $\mathrm{Ba} / \mathrm{Sr}$ & 32 & 4 & 20 & -5 & 37 & 22 & 48 & 39 & 29 & 39 & 15 & -10 & 93 & 10 & 2 & 5 & 7 & -26 & -34 & 13 & 78 & & \\
\hline
\end{tabular}

Note: Correlation coefficients significant at $99.5 \%$ confidence level are shown in boldface type.

for this is that $\mathrm{Sr}$ is strongly associated with one of the major components, $\mathrm{CaCO}_{3}$, in the calcareous oozes which is in effect "diluting", the remaining trace element-bearing component producing a close array effect. It might be suggested of course that a clay component similar in composition to the mean Site 328 clay is the main carrier of trace elements. The mean Site 328 clay is indeed richer in all trace element components apart from $\mathrm{Sr}$ (cf. Table 4). However, there are certain factors which rule against this. The first is that most element plots (Figures 3-8) of Site 328 clay sediments display a wide scatter, whereas equivalent plots for Site 329 sediments give much smoother linear trends which do not necessarily translate directly towards the origin as would be expected from a single dilution process with a relatively inert component such as $\mathrm{CaCO}_{3}$. The second factor is that some elements $(\mathrm{Fe}, \mathrm{Mn}, \mathrm{Ni}, \mathrm{Cr}$, $\mathrm{La}, \mathrm{Ce}, \mathrm{Pb}$, and $\mathrm{Th}$ ) do not have a significantly increased negative correlation with $\mathrm{Sr}$ as might be expected for a simple dilution effect.

The alternative explanations are that either such a clay component differs in composition from Site 328 clay and is much more uniform, or that most of the transition and trace elements (except $\mathrm{Sr}$ ) are associated directly with the siliceous component at Site 329 .

3) There are some major differences in the correlation coefficients of many element pairs between the two sites. To illustrate this the actual differences in correlation coefficient are shown as a matrix in Table 6. Positive values indicate an increase in degree of correlation (whether positive or negative correlation) at Site 329 relative to Site 328 , while negative values indicate a decrease. Only changes which are statistically significant at the $99.5 \%$ confidence level are shown. Some of the most notable changes occur with $\mathrm{Cu}-\mathrm{Sr}, \mathrm{Zr}-\mathrm{Sr}, \mathrm{Zn}-$ $\mathrm{Sr}, \mathrm{Ba}-\mathrm{Sr}, \mathrm{Fe}-\mathrm{Zr}, \mathrm{Fe}-\mathrm{Nb}, \mathrm{Zn}-\mathrm{Nb}, \mathrm{Rb}-\mathrm{Zr}, \mathrm{Fe} / \mathrm{Mn}-\mathrm{Nb}$, $\mathrm{Fe} / \mathrm{Mn}-\mathrm{Zr}, \mathrm{Rb} / \mathrm{Sr}-\mathrm{Fe} / \mathrm{Mn}$, and $\mathrm{Ba} / \mathrm{Sr}-\mathrm{Ba} / \mathrm{Rb}$. Some of these differences are illustrated in Figures 3-8.
4) Iron and manganese have different relationships at the two sites. There is no correlation between $\mathrm{Fe}$ and $\mathrm{Mn}$ in the Site 328 clays, but a significant correlation between $\mathrm{Fe}$ and $\mathrm{Mn}$ at Site 329. Furthermore, the $\mathrm{Fe} / \mathrm{Mn}$ ratio has only a moderate correlation with $\mathrm{Fe}$ in the clays, but a strong negative correlation with $\mathrm{Mn}$, indicating that the latter is the main variable. But at Site $329, \mathrm{Fe} / \mathrm{Mn}$ is very strongly correlated with $\mathrm{Fe}$ and moderately with $\mathrm{Mn}$, indicating that $\mathrm{Fe}$ is the main variable. This suggests differing redox conditions during sedimentation at the two sites.

5) A similar behavior is apparent with $\mathrm{Cr}$ and $\mathrm{Ni}$. The correlation between the two is poor at Site 328, but very strong at Site 329 , while the $\mathrm{Cr} / \mathrm{Ni}$ ratio is strongly correlated with $\mathrm{Cr}$, but not $\mathrm{Ni}$, in the clays, yet strongly correlated with both $\mathrm{Cr}$ and $\mathrm{Ni}$ in the calcareous siliceous oozes.

6) $\mathrm{Mn}$ correlates significantly only with $\mathrm{Ni}$ and $\mathrm{Zn}$ in the deep water site, yet there is a very poor correlation between these three elements, in the shallow water site. This suggests manganese micronodules as a major component governing the distribution of these elements at Site 328 . At Site 329 , on the other hand, there is a strong covariance between $\mathrm{Mn}$ and $\mathrm{Ba}, \mathrm{Ce}, \mathrm{La}$, and $\mathrm{Y}$.

\section{CONCLUSIONS}

Studies by a number of workers on the major and minor element geochemistry of deep-sea sediments have established the overall range of composition of different types of ocean sediment, some areal-, time-, and depth-dependent variations and some interelement relationships (see, for instance, Goldberg and Arrhenius, 1958; El Wakeel and Riley, 1961; Donnelly and Nalli, 1972; Pimm, 1974; Marchig and Vallier, 1974; Fleet and Kempe, 1974; Cook, 1974). There is also considerable data on oceanic iron-rich basal sediments (see review by Bonatti, 1975). Rather than duplicate these studies, the present investigation has 


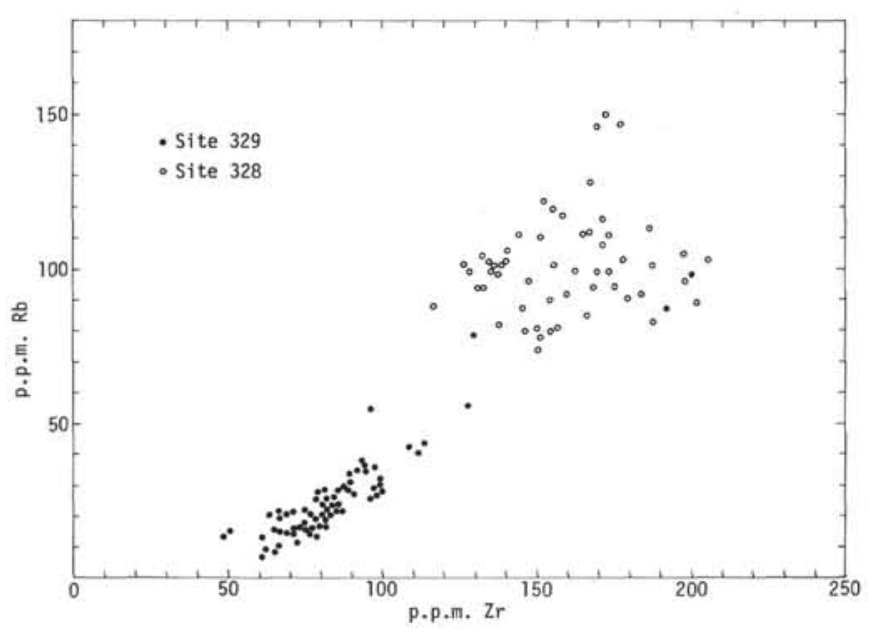

Figure 3. $R b$ versus $Z r$ for Site 328 and Site 329 sediments.

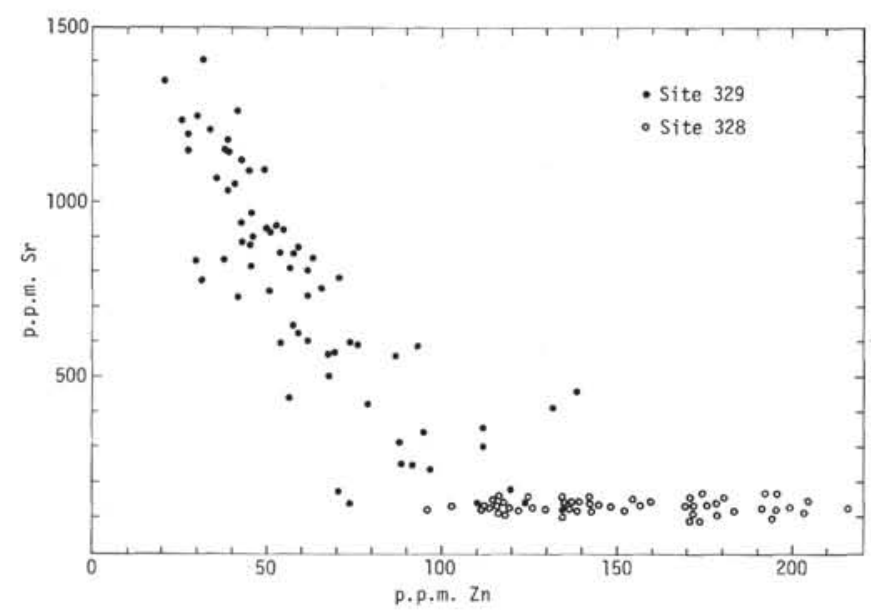

Figure 4. Sr versus Zn for Site 328 and Site 329 sediments.

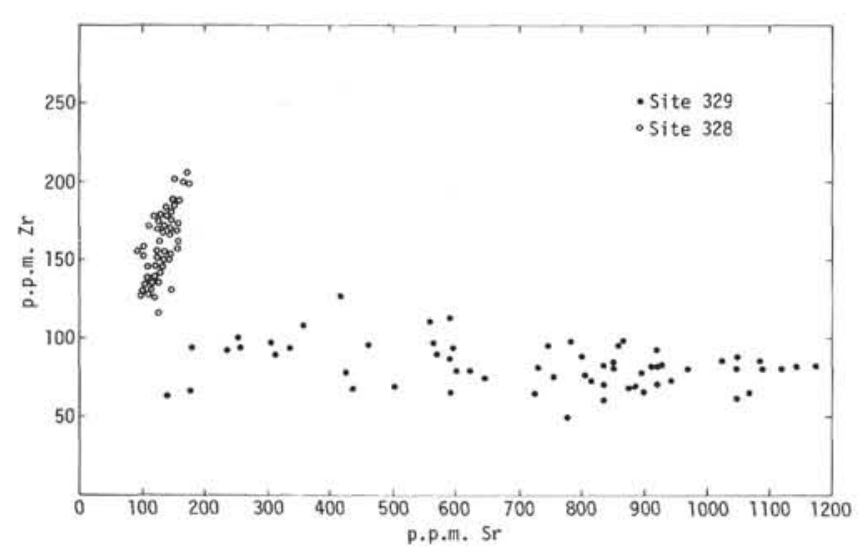

Figure 5. Zr versus Sr for Site 328 and Site 329 sediments.

concentrated on providing precise minor and trace element data on a large number of samples from two adjacent sites of differing bathymetry, but equivalent agerange, with the object of establishing trace element behavior patterns under differing conditions of abyssal sedimentation.

It is clear that there are very substantial differences in trace element abundance levels and interelement relationships at the two sites. At Site 329 , on the Falkland Plateau, the sequence is dominated by variations in proportion of the two major biogenous components, calcareous coccoliths, and siliceous diatoms. Most of the trace elements, with the exception of $\mathrm{Sr}$, are associated with the siliceous component and there is strong covariance between many of the trace elements. In the deep water clay successions of Site 328 in the Falkland Outer Basin, there is much less coherence in trace element behavior, which may reflect differences in the mineralogy and source of the sediments, and (in the case of $\mathrm{Fe}-\mathrm{Mn}$ ) with differing $\mathrm{Eh}$ conditions during deposition or diagenesis. The differing trace element relationships indicate that clay, equivalent in composition to that at Site 328 , is not primarily acting as a carrier for trace elements in the siliceous component at Site 329, but suggests either that trace elements are directly associated with the siliceous component, or that any clay component is different in composition to that at Site 328.

Although the two sites are not very far apart, it appears that the sediment source was very different. In a paleogeographic context, by the time the sediments under consideration were being deposited, Africa had effectively cleared the eastern end of the Falkland Plateau and the proto-Atlantic was established. The

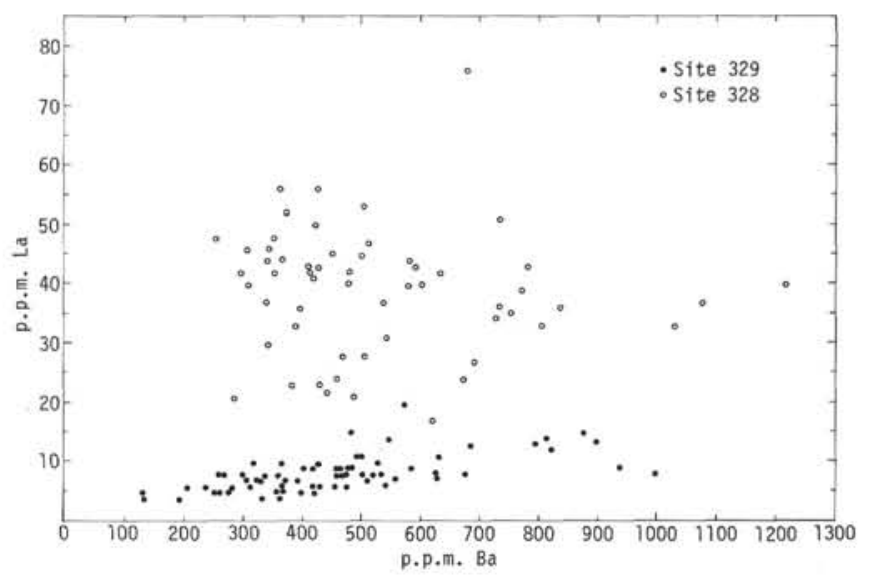

Figure 6. La versus Ba for Site 328 and Site 329 sediments.

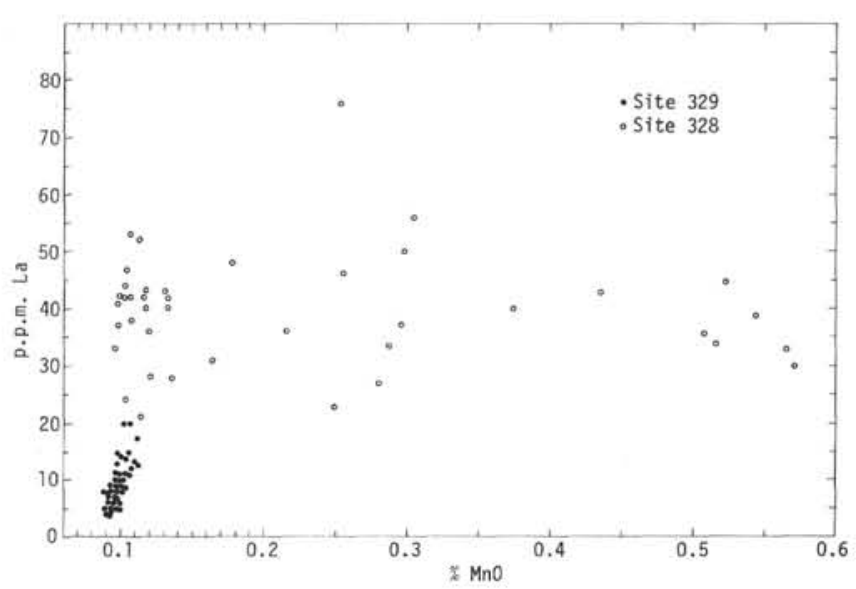

Figure 7. La versus Mn for Site 328 and Site 329 sediments. 
TABLE 6

Matrix Showing the Difference in the Correlation Coefficients for Element Pairs Between Sites 328 and 329

\begin{tabular}{|c|c|c|c|c|c|c|c|c|c|c|c|c|c|c|c|c|c|c|c|c|c|}
\hline & $\mathrm{Ti}$ & $\mathrm{Fe}$ & $\mathrm{Mn}$ & $C_{r}$ & $\mathrm{Ni}$ & $\mathrm{Cu}$ & $\mathrm{Zn}$ & $\mathrm{Rb}$ & $\mathrm{Sr}$ & $Y$ & $\mathrm{Zr}$ & $\mathrm{Nb}$ & $\mathrm{Ba}$ & $\mathrm{La}$ & $\mathrm{Ce}$ & $\mathrm{Pb}$ & Th & $\mathrm{Cr} / \mathrm{Ni}$ & $\mathrm{Fe} / \mathrm{Mn}$ & $\mathrm{Rb} / \mathrm{Sr}$ & $\mathrm{Ba} / \mathrm{Rb}$ \\
\hline $\mathrm{Fe}$ & i. & & & & & & & & & & & & & & & & & & & & \\
\hline $\mathrm{Mn}$ & 0.44 & 0.49 & & & & & & & & & & & & & & & & & & & \\
\hline $\mathrm{Cr}$ & 0.79 & 0.58 & - & & & & & & & & & & & & & & & & & & \\
\hline $\mathrm{Ni}$ & 0.48 & 0.44 & -0.43 & 0.73 & & & & & & & & & & & & & & & & & \\
\hline $\mathrm{Cu}$ & - & - & - & - & & & & & & & & & & & & & & & & & \\
\hline $\mathrm{Zn}$ & - & 0.55 & - & 0.52 & - & 53 & & & & & & & & & & & & & & & \\
\hline $\mathrm{Rb}$ & 0.38 & 0.44 & - & 0.38 & - & $\overline{26}$ & - & & & & & & & & & & & & & & \\
\hline $\begin{array}{l}\text { Sr } \\
Y\end{array}$ & 0.43 & 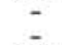 & $0 . \overline{62}$ & $\overline{0.49}$ & $\overline{0.47}$ & & 0.98 & 0.51 & - & & & & & & & & & & & & \\
\hline $\begin{array}{l}\mathrm{Y} \\
\mathrm{Zr}\end{array}$ & 0.60 & 0.97 & $\begin{array}{c}0.62 \\
-\end{array}$ & $\begin{array}{l}0.49 \\
-\end{array}$ & $\begin{array}{l}0.47 \\
-\end{array}$ & - & 0.36 & $0 . \overline{80}$ & 0.91 & - & & & & & & & & & & & \\
\hline $\mathrm{Nb}$ & 0.42 & 0.65 & - & - & - & - & 0.54 & 0.83 & 0.68 & - & - & & & & & & & & & & \\
\hline $\mathrm{Ba}$ & 0.35 & - & 0.35 & 0.40 & - & 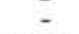 & - & - & -0.95 & 0.41 & - & - & & & & & & & & & \\
\hline $\mathrm{La}$ & - & - & 0,80 & - & - & -0.45 & 0.42 & - & - & - & 0.45 & - & 0.67 & & & & & & & & \\
\hline $\mathrm{Ce}$ & - & - & 0.75 & - & - & -0.40 & - & - & - & - & 0.35 & - & 0.64 & - & & & & & & & \\
\hline $\mathrm{Pb}$ & - & - & 0.36 & - & - & - & - & - & - & - & 0.54 & 0.37 & - & - & - & - & & & & & \\
\hline Th & - & - & - & - & - & - & - & - & - & - & & - & - & - & - & - & - & & & & \\
\hline $\mathrm{Cr} / \mathrm{Ni}$ & 0.95 & 0.89 & -0.62 & - & 0.85 & - & 0.99 & 0.91 & - & 0.54 & 0.95 & 0.62 & - & - & - & - & - & & & & \\
\hline $\mathrm{Te} / \mathrm{Mn}$ & 0.69 & 0.61 & -1.03 & - & -0.93 & - & -1.15 & 1.07 & - & 0.64 & 1.09 & 0.52 & - & 0.43 & - & 0.42 & - & - & & & \\
\hline $\mathrm{Rb} /$ & - & - & - & - & - & -0.60 & 0.38 & - & - & - & & 0.88 & - & - & - & - & -0.37 & 0.66 & 0.77 & & \\
\hline $\mathrm{Ba} / \mathrm{Rb}$ & - & - & - & - & - & -0.76 & - & - & -0.37 & - & 0.65 & - & - & - & - & - & - & - & - & - & - \\
\hline $\mathrm{Ba} / \mathrm{Sr}_{\mathrm{r}}$ & 0.38 & 0.60 & - & - & - & - & 0.35 & 0.38 & 1.08 & - & 0.48 . & 0.74 & -0.46 & $0.5 \xi$ & 0.40 & 0.42 & - & 0.78 & 0.97 & 0.73 & -0.92 \\
\hline
\end{tabular}

Note: Positive values indicate an increase in correlation coefficient (whether positive or negative correlation) in Site 329 sediments while negative values indicate a decrease in degree of correlation. Only changes significant at better than $99.5 \%$ confidence level, on the basis of student $T$ test, are included.

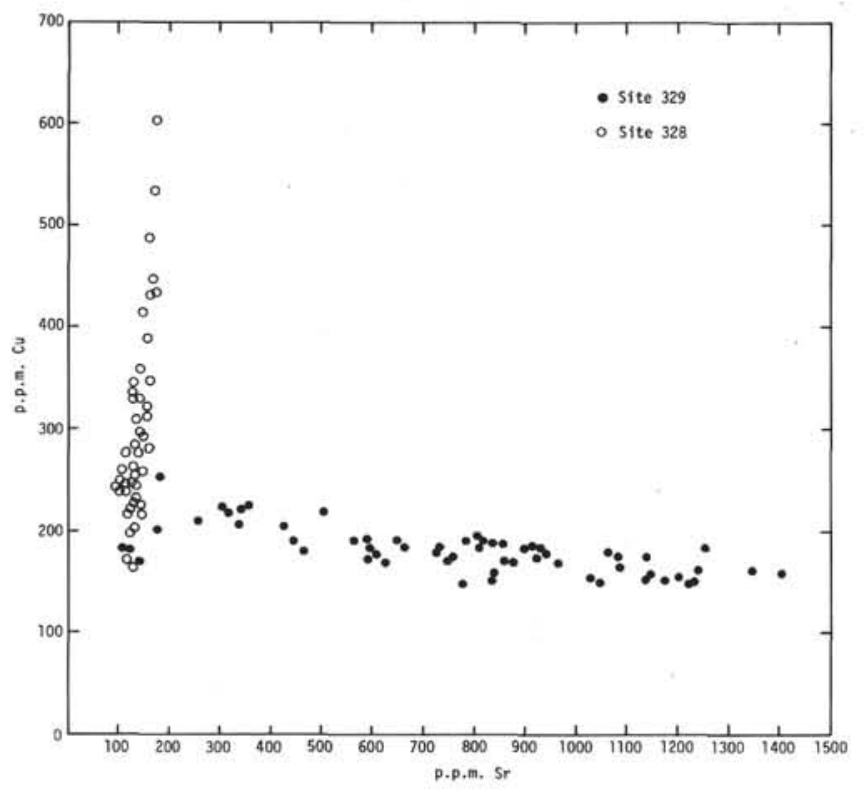

Figure 8. Cu versus Sr for Site 328 and Site 329 sediments.

situation of Site 328 allows the possibility of sediments being derived from either the Antarctic continent, or from southernmost South America via the long narrow Falkland Trough. In either case transport may have been largely through the agency of bottom currents. The downhole variability of Site 328 sediments may reflect this variable sediment source. On the other hand, if transport of terrigenous clay components was largely by bottom currents, it is unlikely that much of the clay would find its way onto the shelf environment of the Falkland Plateau. This would concur with the geochemical findings.

Finally, short-, medium-, and long-term trends are evident in the downhole geochemical patterns. While some of these trends can be correlated directly with changing lithology and breaks in sedimentation, the significance of other trends is more obscure and may be related to variations in sediment source, gradual changes in the nature of biogenic components, or to varying $p \mathrm{H}$ and $\mathrm{Eh}$ conditions connected with changes in bottom-current activity.

\section{REFERENCES}

Bonatti, E., 1975. Metallogeneris at oceanic spreading centers: Ann. Rev. Earth Planet. Sci., v. 3, p. 401-431.

Cook, P.J., 1974. Major and trace element geochemistry of sediments from Deep Sea Drilling Project, Leg 27, Sites 259-263, Eastern Indian Ocean. In Heirtzler, J., Veevers, J., et al., Initial Reports of the Deep Sea Drilling Project, Volume 27: Washington (U.S. Government Printing Office), p. 481-497.

Donnelly, T.W. and Nalli, G., 1972. Mineralogy and chemistry of Caribbean sediments. In Edgar, N.T., Saunders, J.B., et al., Initial Reports of the Deep Sea Drilling Project, Volume 15: Washington (U.S. Government Printing Office), p. 929-946.

El-Wakeel, S.K. and Riley, J.P., 1961. Chemical and mineralogical studies of deep sea sediments: Geochim. Cosmochim. Acta, v. 25, p. 110-146.

Fleet, A.J. and Kempe, D.R.C., 1974. Preliminary geochemical studies of the sediments from DSDP Leg 26, Southern Indian Ocean. In Davies, T.A., Luyendyk, B., et al., Initial Reports of the Deep Sea Drilling Project, Volume 26: Washington (U.S. Government Printing Office), p. 541-551.

Goldberg, E.D. and Arrhenius, G., 1958. Chemistry of Pacific pelagic sediments: Geochim. Cosmochim. Acta, v. 13, p. 153-212.

Marchig, V. and Vallier, T.L., 1974. Geochemical studies of sediment and interstitial water, Sites 248 and 249, Leg 25, Deep Sea Drilling Project. In Simpson E.S.W., Schlich, R., et al., Initial Reports of the Deep Sea Drilling Project, Volume 25: Washington (U.S. Government Printing Office), p. 405-415.

Pimm, A.C., 1974. Mineralization and trace element variation in deep-sea pelagic sediments of the Wharton Basin, Indian Ocean. In von der Borch, C.C., Sclater, J.G., et al., Initial Reports of the Deep Sea Drilling Project, Volume 22: Washington (U.S. Government Printing Office), p. $469-476$. 